\title{
The CD95/CD95L Signaling Pathway: A Role in Carcinogenesis
}

\author{
Amélie Fouqué and Patrick Legembre
}

\section{Contents}

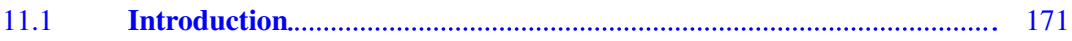

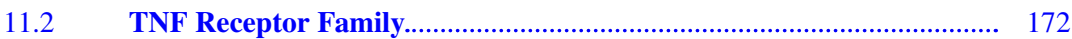

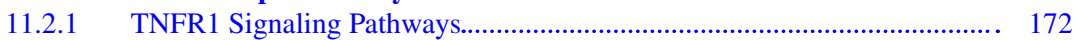

11.2.2 TNF/TNFR: A Gold Mine for Therapeutic Tools........................................ 173

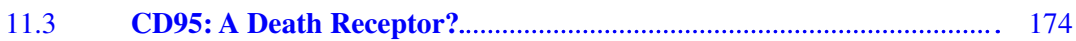

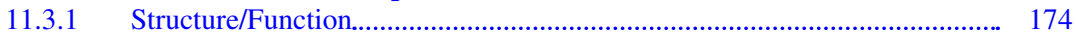

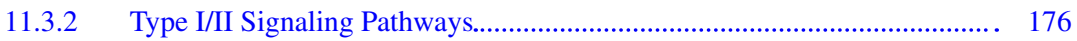

11.3.3 What Can We Learn from CD95 Mutations?................................................ 177

11.3.4 Regulation of the Initial Steps of CD95-Mediated Signaling...................... 178

11.3.4.1 Lipid Rafts................................................................................................ 178

11.3.4.2 Posttranslational Modifications..................................................................... 178

11.3.4.3 CD95 Internalization.................................................................................. 179

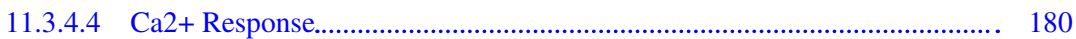

11.3.5 Programmed Necrosis also known as Necroptosis....................................... 180

11.3.6 CD95L, an Inflammatory/Oncogenic Cytokine?......................................... 180

11.3.6.1 A Ligand to Create Immune Privileges.............................................................. 180

11.3.6.2 At Least Two Different Ligands and Two Different Signals.......................... 181

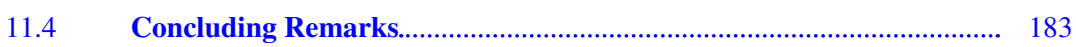

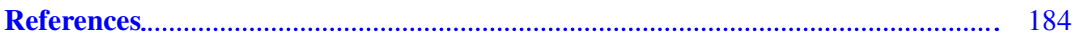

A. Fouqué

Université de Rennes-1, Rennes, France

INSERM U1242, Equipe Labellisée Ligue Contre Le Cancer, Rennes, France

P. Legembre $(\bowtie)$

Université de Rennes-1, Rennes, France

INSERM U1242, Equipe Labellisée Ligue Contre Le Cancer, Rennes, France

CLCC Centre Eugéne Marquis, Rennes, France e-mail: patrick.legembre@inserm.fr

\subsection{Introduction}

Apoptosis, or programmed cell death, plays a pivotal role in development, organ homeostasis, and immunosurveillance. The term apoptosis was coined by Kerr et al. in 1972 [1] to describe the process of cell death associated with morphological changes, including nucleus and cytoplasm condensation and protuberances from the plasma membrane producing apoptotic bodies, so-called blebs, which are rapidly phagocytosed [1, 2]. 
Inhibition of this cellular process is observed in different pathologies, such as cancer and autoimmunity, while amplification of the apoptotic signal was reported in neurodegenerative disorders including Alzheimer's and Parkinson's diseases $[3,4]$, as well as infection by human immunodeficiency virus (HIV).

The origin of the apoptotic signal has been used to distinguish two main signaling pathways. The intrinsic pathway stems from accumulation of DNA damage, deregulation of mitochondrial function, or virus infection and induces the release of proapoptotic factors from the mitochondria, whereas extrinsic signals are transmitted by the binding of apoptotic ligands to death receptors present at the cell surface. Interconnections exist between these two signaling pathways: both leading to the activation of a family of cysteine proteases specific for aspartic acid residues, called caspases [5]. The apoptotic role of mitochondria is associated with reduction in its transmembrane potential and the loss of its extracellular membrane integrity, leading to the release of different apoptogenic factors in the cytosol. Among them, cytochrome c associates with the caspase-9/APAF-1 complex to form the apoptosome and trigger apoptosis [6].

These two signaling pathways share common features, and both require the aggregation of initiator caspases as their preliminary events. During interactions with respective ligands, members of the death receptor superfamily recruit adaptor proteins such as Fas-associated protein with a death domain (FADD) [7, 8] or tumor necrosis factor (TNF) receptor 1-associated death domain protein (TRADD) [9], resulting in the aggregation and activation of the initiators caspase- 8 and caspase-10 to form the death-inducing signaling complex (DISC) [10]. In a similar manner, release of cytochrome $\mathrm{c}$ and ATP by mitochondria promotes the formation of the apoptosome with the cytosolic APAF-1, thereby aggregating and activating the initiator caspase-9, which in turn cleaves caspase-3 [11].

It should be kept in mind that death receptors CD95 [12], TNFR1 [13], DR4 [14], DR5 [15], and DR6 [16] have been cloned based on their ability to elicit apoptosis. Although studies have revealed the ability of Fas/CD95, DR4, and DR5 in triggering non-apoptotic signaling pathways even immediately after their cloning [17, 18], most, if not all, studies have been focused on characterizing the molecular events leading to cell death. Accordingly, several agonistic molecules were developed in order to kill cancer cells, neglecting the impact of non-apoptotic signals in pathophysiological contexts. More recent data changed this vision by evaluating the biological role of death receptor-mediated non-apoptotic signaling pathways in chronic inflammatory disorders and carcinogenesis.

In this chapter, apoptotic signaling pathways induced by death receptors are discussed. Moreover, recent evidences pointing to the non-apoptotic signals transmitted by the same receptors are brought up, which may imply their tremendous impact on tumor progression and the design of therapeutic tools.

\subsection{TNF Receptor Family}

Death receptors TNFR1, Fas, DR3, DR4, DR5, and DR6 belong to the tumor necrosis factor receptor (TNFR) superfamily. These type I transmembrane proteins share common features, such as extracellular amino-terminal cysteine-rich domains (CRDs) [19, 20], which contribute to ligand specificity [21], and pre-association of the receptor at the plasma membrane [22-24] and a conserved 80-amino acid sequence located in their cytoplasmic tail called death domain (DD), which is necessary for DISC formation and initiation of the apoptotic signal $[25,26]$.

\subsubsection{TNFR1 Signaling Pathways}

TNF- $\alpha$ exerts its effects by binding to two receptors, TNFR1 and TNFR2 [20]. Recently, progranulin was identified as a ligand of TNFR with a higher affinity than TNF- $\alpha$. Progranulin antagonizes TNF- $\alpha$ signaling and plays a critical role in the pathogenesis of inflammatory arthritis in mice [27]. TNFR1, a $55 \mathrm{kDa}$ protein expressed in almost all cell types, presents a DD in its 
intracellular region, whereas TNFR2, a $75 \mathrm{kDa}$ protein, is mainly detected in oligodendrocytes, astrocytes, T-cells, myocytes, thymocytes, endothelial cells, and human mesenchymal stem cells [28]. Uncertainty remains on the TNFR2 signaling pathway, which has been previously reviewed [28]. The CRD1 of CD95, TNFR1, and TNFR2 is involved in homotypic interactions, leading to pre-association of the receptor as a homotrimer in the absence of ligand [23, 24, 29]. This domain has been designated as the pre-ligand binding assembly domain (PLAD) [29]. Receptors of the TNFR superfamily do not possess any enzymatic activity on their own and rely on the recruitment of adaptor proteins for signaling. Among these adaptor proteins, TRADD or FADD is instrumental in the implementation of cell death processes [7-10].

TNF is synthesized as a $26 \mathrm{kDa}$ transmembrane type II protein (m-TNF) of 233 amino acids [30] which can be cleaved by the metalloprotease TACE $[31,32]$ to release the $17 \mathrm{kDa}$ soluble form of the cytokine (cl-TNF). In contrast to cl-TNF, which only activates TNFR1, m-TNF can bind and activate both TNFR1 and TNFR2 [33].

Activation of TNFR1 leads to the induction of cellular processes ranging from cell death (apoptosis or necroptosis) to cell proliferation, migration, and differentiation; the implementation of such different cellular responses reflects the formation of different molecular complexes after receptor activation [28]. Binding of TNF to TNFR1 causes the formation of two consecutive complexes. While the plasma membrane complex (complex I) elicits a non-apoptotic signaling pathway, a second, internalized complex (complex II or DISC) triggers cell death [2]. In the presence of TNF, the adaptor protein TRADD interacts with TNFR1 and recruits other proteins involved in the signaling of the receptor, such as TRAF2, cIAP1, cIAP2, and RIP1, to form complex I. At the plasma membrane, this complex activates the NF- $\mathrm{KB}$ signaling pathway, which in turn promotes the transcription of antiapoptotic genes such as cIAP1, cIAP2, and c-FLIP [34]. The linear ubiquitin chain assembly complex (LUBAC) is also recruited to complex I via cIAP-generated ubiquitin chains [35]. HOIL-1,
HOIP, and sharpin constitute the LUBAC complex. HOIL-1 and HOIP add a linear ubiquitin chain by catalyzing the head-to-tail ligation of ubiquitin [36] to RIP1 and NEMO (IKK- $\gamma$ ) in complex I [37], thereby activating NF-кB.

TNF-induced caspase activation is mediated by a second, intracellular complex II, which is formed when complex I dissociates from the receptor, along with FADD and caspase-8 recruitment [2]. NF- $\kappa \mathrm{B}$ activation leads to c-FLIP overexpression, preventing formation of complex II. Contrariwise, when NF- $\mathrm{BB}$ activation is blocked, c-FLIP, whose protein half-life is short [38], is absent, and cells experience death [2]. RIP1 is deubiquitinated by enzymes such as Cezanne [39] and CYLD [40], and the complex composed of TRADD and RIP1 moves to the cytosol to form complex II. FADD is recruited to TRADD by DD-DD interaction and binds caspase-8 [2]. Noteworthy, when caspase- 8 activity is inhibited or its expression is extinguished, DISC is unable to trigger the apoptotic signaling pathway; but TNFR1 or CD95 stimulation leads to the activation of another cell death signal, namely, necroptosis [41, 42]. To prevent the induction of the necroptotic signal, caspase- 8 cleaves and inactivates RIP1 and RIP3 [43]. The fine-tuned control of necroptosis by members of the apoptotic signaling pathway in the organism has been elegantly confirmed by experiments showing that the embryonic lethality of mice harboring the single $\mathrm{KO}$ of caspase- 8 or FADD is rescued by an additional $\mathrm{KO}$ of the RIP3 gene [44-46].

\subsubsection{TNF/TNFR: A Gold Mine for Therapeutic Tools}

Many studies on TNF demonstrated its pivotal role in fueling inflammation, a multistep process that promotes autoimmunity (e.g., rheumatoid arthritis, ankylosing spondylitis, Crohn's disease, psoriasis, and refractory asthma) and cancer. Many TNF inhibitors, such as neutralizing monoclonal antibodies (mAbs) (e.g., infliximab, adalimumab, and golimumab), have been developed to treat these chronic inflammatory disorders, 
demonstrating that altering ligand/receptor interactions with neutralizing mAbs is an invaluable opportunity to treat certain chronic inflammatory disorders. Other TNF- $\alpha$ antagonists, such as etanercept, a TNFR2-immunoglobulin Fc fusion protein, can improve the clinical course of rheumatoid arthritis [47].

While findings accumulate to decipher the molecular mechanisms involved in the induction of apoptotic and non-apoptotic signaling pathways by TNFR1 and to elucidate how the receptor can switch from one signal to the other, the mechanistic links involved in the implementation of non-apoptotic signaling pathways by CD95 remain elusive. However, recent findings have revealed its proinflammatory effects [48-54].

\subsection{CD95: A Death Receptor?}

In 1989, identification of the mAb APO-1 by Peter Krammer et al. revealed the existence of a $52 \mathrm{kDa}$ protein whose aggregation was able to transmit an apoptotic signal in cancer cells [55]. This receptor was identified in 1991 by Nagata and colleagues and called Fas (CD95 or APO-1) [12]. Its ligand, FasL, was cloned in 1993 by the same group and was found to be mainly expressed at the surface of activated T-lymphocytes [56] and natural killer (NK) cells [57]; however, its expression was also detected in different tissues in which the presence of acute or chronic inflammation is undesirable including the eyes [58] and testes [59]. In addition, two mouse models, in which either the level of CD95 expression was downregulated (due to an insertion of a retrotransposon in intron 2 of the receptor gene, these mice are called lymphoproliferation (Lpr)
[60-62]) or the CD95L affinity for CD95 was reduced (due to the germ line mutation F273L in CD95L, called generalized lymphoproliferative disease ( gld), which decreases CD95L binding to CD95 [63, 64]), have provided some insight into the pivotal role played by this interaction in immunosurveillance and immune tolerance [65].

\subsubsection{Structure/Function}

The CD95 gene (APT-1) consists of nine exons, with exon 6 encoding the transmembrane domain [66] (Fig. 11.1). CD95 can be resolved under denaturing conditions between 40 and $50 \mathrm{kDa}$ by SDS-PAGE. The receptor is a type I transmembrane protein harboring three CRDs. Similar to the TNF receptor [29], CD95 is pre-associated at the plasma membrane as a homotrimer, and this quaternary structure is mandatory for transmission of the apoptotic signals in the presence of CD95L [23, 24]. Homotrimerization of CD95 occurs mainly through homotypic interactions of the CD95-CRD1 [22-24]. Binding of CD95L or agonistic anti-CD95 mAbs to CD95 alters both the conformation and the extent to which the receptor is multimerized at the plasma membrane. The intracellular region of CD95 encompasses an 80-amino acid stretch designated as the DD (Fig. 11.1), which consists of six antiparallel $\alpha$-helices [67]. Upon addition of CD95L, CD95 undergoes conformational modification of its DD, which induces a shift of helix 6 and fusion with helix 5, promoting both oligomerization of the receptor and recruitment of the adaptor protein FADD [68]. A consequence of the opening of the globular structure of CD95 is that the receptor becomes connected through this bridge, which

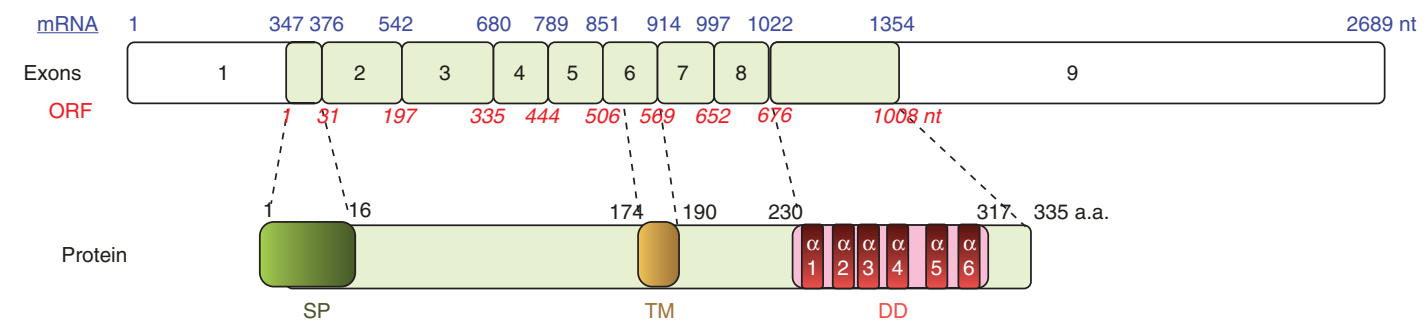

Fig. 11.1 CD95: mRNA to protein 
increases the magnitude of its homo-aggregation. This long helix allows the stabilization of the complex by recruiting FADD. Overall, the CD95-DD/FADD-DD crystal structure provides some insights into the formation of the large CD95 clusters observed using imaging or biochemical methods in cells stimulated with CD95L. In addition, it also confirms that alteration in the CD95 conformation plays an instrumental role during signal induction [68]. However, this elongated C-terminal $\alpha$-helix favoring the cis-dimerization of CD95-DD was challenged by Driscoll et al. who did not observe the fusion of the last two helices at a more neutral $\mathrm{pH}(\mathrm{pH}$ 6.2), compared to the acidic condition $(\mathrm{pH} 4)$ used in the initial study to resolve the CD95-DD/FADD-DD structure [68]. Consequently, at pH 6.2, association of CD95 with FADD predominantly consisted of a 5:5 complex, which occurred via a polymerization mechanism involving three types of asymmetric interactions but without major alteration of the DD globular structure $[69,70]$. It is likely that the low $\mathrm{pH}$ condition used in the study performed by Scott et al. altered CD95 conformation and resulted in the formation of nonphysiological CD95/FADD oligomers [68]. Nonetheless, it cannot be excluded that a local decrease in the intracellular $\mathrm{pH}$ affects the initial steps of the CD95 signaling pathway in vivo, through promoting the opening of the CD95-DD and eventually contributing to the formation of a complex eliciting a sequence of events different from the one occurring at physiologic $\mathrm{pH}$.

Once docked on CD95-DD, FADD selfassociates [71] and binds procaspase-8 and procaspase-10, which are auto-processed and released in the cytosol as active caspases, which cleave many substrates leading to the execution of the apoptotic program and cell death. The complex CD95/FADD/caspase-8/ caspase-10 is called DISC (Fig. 11.2) [10]. Due to the importance of DISC formation in

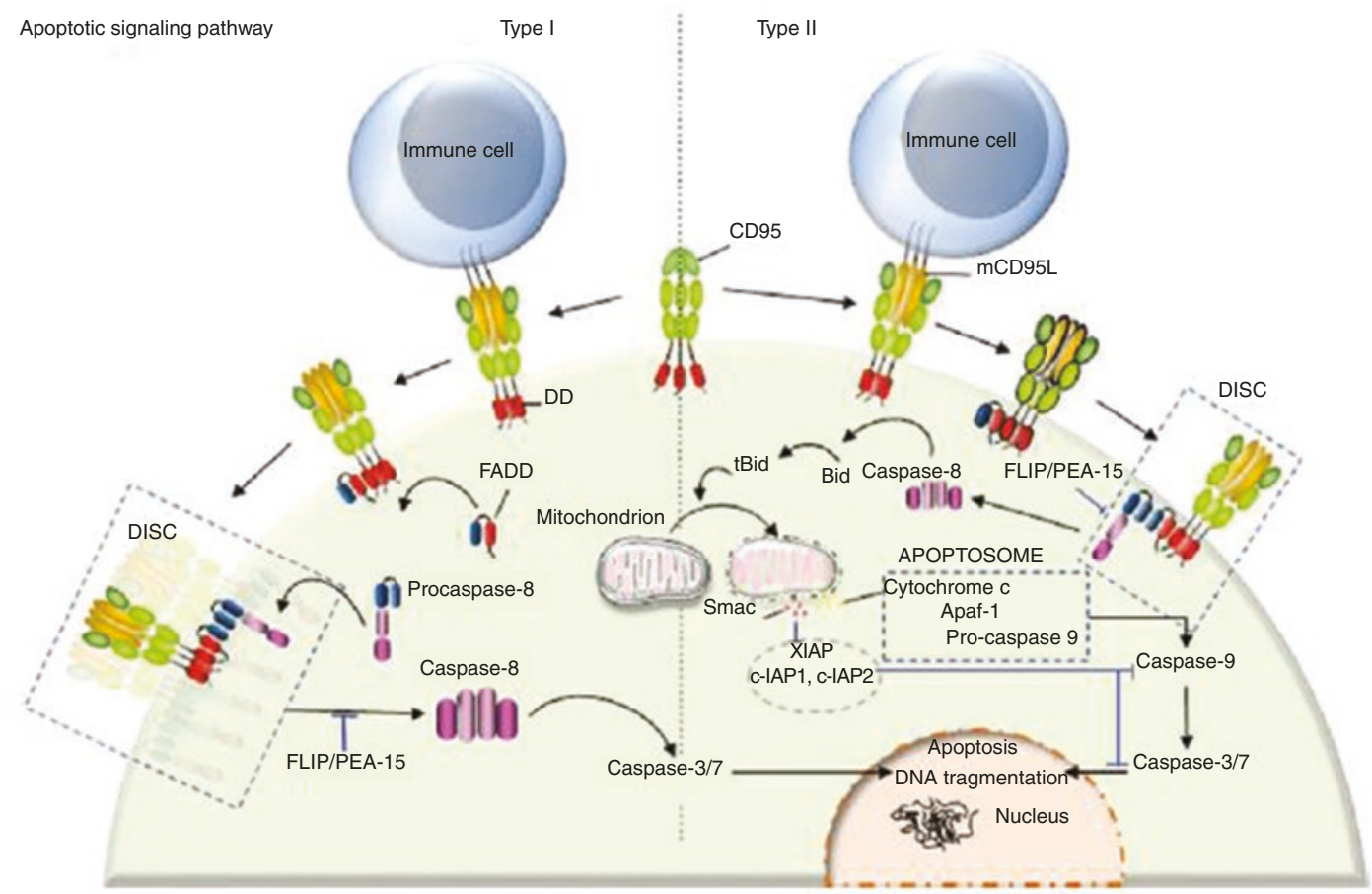

Fig. 11.2 Type I/II cells. Binding of transmembrane CD95L to CD95 leads to DISC formation. DISC consists of FADD and procaspase-8. c-FLIP and PEA-15 bind to FADD and prevent caspase- 8 recruitment. At the DISC level, aggregation of procaspase- 8 promotes its autocleavage and activation. Cleaved caspase- 8 is then released in the cytosol where it promotes the cascade of caspase activation leading to apoptosis. Type I cells are characterized by an efficient DISC formation, which releases sufficient caspase- 8 to directly activate caspase- 3 . By contrast, type II cells present a weak DISC formation, and the low amount of released caspase- 8 activates the mitochondrion-dependent apoptotic pathway to amplify death signal 
the fate of cells, it is not surprising that numerous cellular and viral proteins were reported to hamper the formation of this structure, such as FLIP [72, 73] and PED/PEA-15 [74], which interfere with the recruitment of caspase-8/ caspase-10 (Fig. 11.2).

\subsubsection{Type I/II Signaling Pathways}

Following the discovery of CD95 and the first steps of its signaling pathway, Peter and colleagues described that cells can be divided in two groups with regard to the kinetics through which they respond to CD95-mediated apoptotic signals, the magnitude of DISC formation, and the role played by the mitochondrion in this pathway [75]. DISC formation occurs rapidly and efficiently in type I cells releasing a large amount of activated caspase- 8 in the cytosol, while type II cells have difficulty forming this complex, and the amount of active caspase- 8 is insufficient to directly activate the effectors caspase- 3 and caspase-7 [75]. Nonetheless, type II cells experience cell death upon CD95 engagement and are even more sensitive to the CD95-mediated apoptotic signal compared to type I cells [75-77]. This discrepancy can be partly explained by the fact that the low amount of activated caspase- 8 in type II cells is sufficient to cleave BID, a BH3-only protein, which constitutes the molecular link between caspase- 8 activation and the apoptotic activity of mitochondria. Indeed, after cleavage by caspase- 8 , truncated BID (tBID) translocates to mitochondria, where it triggers the release of proapoptotic factors (Fig. 11.2) [78, 79]. Although CD95 stimulation activates the mitochondrion-dependent apoptotic signal in type I and type II cells, it seems that only type II cells are addicted to this signal as they display a higher amount of the caspase-3 inhibitor XIAP compared to type I cells [80]. Among the inhibitor of apoptosis protein (IAP) family, XIAP, cIAP1, and cIAP2 inhibit caspase-3, caspase-7 [81, 82], and procaspase-9 [83] activity by direct binding, thereby preventing access to substrates.
Furthermore, XIAP can function as an E3 ligase whose activity is involved in the ubiquitination of active caspase- 3 and its subsequent degradation through the proteasome [84]. To detach XIAP from caspase- 3 and restore the apoptotic signal, cells require the release of SMAC/DIABLO (second mitochondria-derived activator of caspase/ direct IAP-binding protein with low PI) by the mitochondrion [85, 86], explaining why type II cells are more addicted to this organelle compared to type I cells (Fig. 11.2).

To summarize, DISC formation and IAP amount are two cellular markers allowing a clear discrimination between type I and type II cells. Even though IAP overexpression can account for the mitochondrion dependency observed in type II cells, it remains unclear why DISC formation is hampered in type II cells and/or enhanced in their type I counterparts. Recently, high activity of the lipid kinase phosphoinositide 3-kinase (PI3K) or downregulation of its neutralizing phosphatase, phosphatase and tensin homologue on chromosome 10 (PTEN), was found in type II cells, while this signal is blocked in type I cell lines $[87,88]$. The PI3K signaling pathway was reported to prevent the aggregation of CD95 [89], probably by retaining the receptor outside of lipid rafts [87, 90]. PEA-15, also known as PED, is a protein containing a death effector domain (DED) that has been shown to inhibit the CD95 and TNFR1 apoptotic signals (Fig. 11.2) [74]. Activation of PI3K and its downstream effector, serine-threonine kinase Akt, leads to phosphorylation of PEA-15 at serine 116 [87, 90]; this posttranslational modification promotes its interaction with FADD, ultimately inhibiting DISC formation [91, 92].

Notably, the existence of type I and type II cells is not only an in vitro observation, but has been identified physiologically in the human body. CD95-mediated apoptotic signal cannot be altered in thymocytes or activated T-cells expressing a Bcl-2 transgene, conferring to their type I nature [93], whereas hepatocytes expressing the same transgene resist CD95-induced apoptosis and thus behave as type II cells [94, 95]. 


\subsubsection{What Can We Learn from CD95 Mutations?}

Germinal mutations in APT- 1 have been reported in patients developing a syndrome termed autoimmune lymphoproliferative syndrome type Ia (ALPS, also called Canale-Smith syndrome) [96-98]. ALPS patients show chronic lymphadenopathy and splenomegaly, expanded populations of double-negative $\alpha / \beta$-T-lymphocytes $\left(\mathrm{CD}^{+}{ }^{+} \mathrm{CD} 4^{-} \mathrm{CD}^{-}\right)$, and often develop autoimmunity $[96,97,99,100]$. In agreement with the notion that CD95 behaves as a tumor suppressor, ALPS patients display an increased risk of Hodgkin and non-Hodgkin lymphoma [101]. Predominance of post-germinal center (GC) lymphomas in patients exhibiting either germ line or somatic CD95 mutations can be explained by the fact that, inside germinal centers of the secondary lymphoid follicles, the CD95 signal plays a pivotal role in the deletion of self-reactive maturating B-lymphocytes [102], in addition to the fact that APT-1 belongs to a set of rare genes (i.e., PIM1, c-myc, PAX5, RhoH/TTF, and Bcl-6) subject to somatic hypermutation [103, 104], which may affect biological function. In addition to post-GC lymphomas, significant amounts of mutations in the CD95 gene were found in tumors of various histological origins (reviewed in [54]). Extensive analysis of CD95 mutations and their distribution in $A P T-1$ reveals that, with some exceptions, most are gathered in exons 8 and 9 encoding the CD95 intracellular region (Fig. 11.3) [105]. Remarkably, most of these mutations are heterozygous, mainly localized in CD95-DD, and lead to inhibition of the CD95-mediated apoptotic signal. Indeed, in agreement with the notion that CD95 is expressed at the plasma membrane as

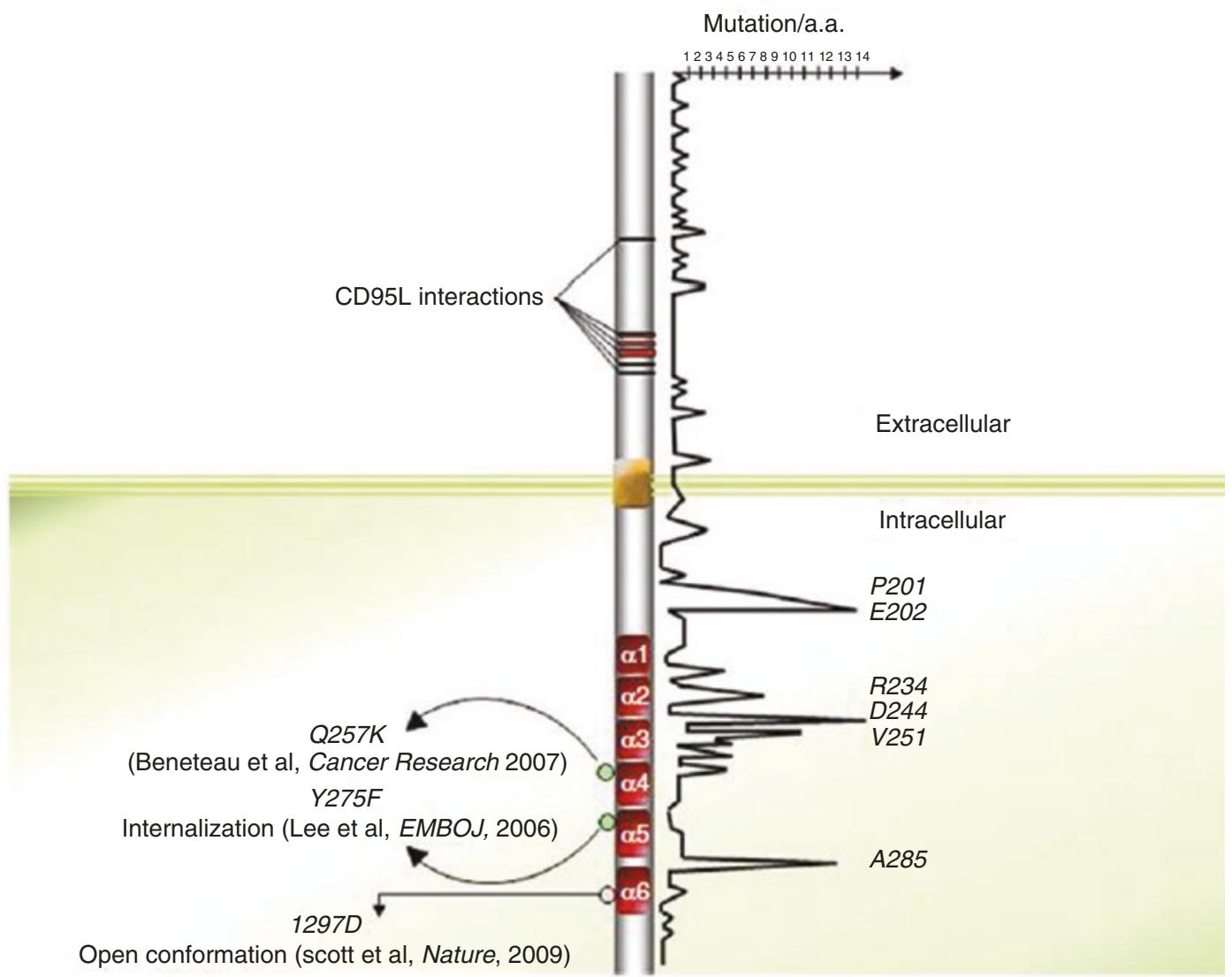

Fig. 11.3 Distribution of somatic and germinal mutations within CD95 protein sequence 
a pre-associated homotrimer [23, 24], formation of heterocomplexes containing wild-type and mutated CD95 prevents FADD recruitment and abrogates the ignition of the apoptotic signal in a dominant manner.

Extensive analysis and positioning of various CD95 mutations described in the literature seem to highlight mutation "hot spots" in the CD95 sequence (Fig. 11.3). Among these hot spots, arginine 234 , aspartic acid 244 , and valine 251 account for a significant amount of the documented CD95 mutations. Indeed, among the 189 mutations annotated in the 335 amino acids of CD95, $30(\sim 16 \%)$ are localized on these three amino acids (Fig. 11.3). Strikingly, the pivotal role played by these amino acids in stabilization or formation of intra- and interbridges between CD95 and FADD may explain these hot spots. For instance, both R234 and D244 contribute to the homotypic aggregation of the receptor and FADD recruitment [67]. Nevertheless, the observation of death domain hot spots is in contradiction with the study of Scott and colleagues demonstrating that the region of the CD95-DD interacting with the FADD-DD extends over a disperse surface through weak binding affinity [68].

Most ALPS type Ia patients affected by malignancies do not undergo loss of heterozygosity (LOH), which formed the hypothesis that preservation of a wild-type allele may contribute to carcinogenesis $[106,107]$. In the same line, it was demonstrated that expression of a unique mutated CD95 allele blocks the induction of apoptotic signals, while it fails to prevent nonapoptotic signals such as NF-кB and MAPK $[106,107]$, whose induction promotes invasiveness in tumor cells [105, 108]. In addition, mutations found in the intracellular CD95-DD exhibit a higher penetrance of ALPS phenotype features in mutation-bearing relatives compared to extracellular mutations. These results suggest that unlike DD mutations, CD95 mutations localized outside the DD somehow prevent the apoptotic signal but may fail to promote non-apoptotic pathways, which may contribute to disease aggressiveness.

\subsubsection{Regulation of the Initial Steps of CD95-Mediated Signaling}

\subsubsection{Lipid Rafts}

In addition to CD95 downregulation or expression of the mutated allele of the receptor, the plasma membrane distribution of CD95 represents an additional pathway for tumor cells to develop resistance to CD95L-expressing immune cells. Indeed, the plasma membrane is a heterogeneous lipid bilayer comprising compacted or liquid-ordered domains, called microdomains, lipid rafts, or detergent-resistant microdomains (DRMs). These domains are described as floating in a more fluid or liquid-disordered 2D lipid bilayer and are enriched in ceramides [109]. It has been elegantly shown that while CD95 is mostly excluded from lipid rafts in activated T-lymphocytes, TCR-dependent reactivation of these cells leads to rapid distribution of the death receptor into lipid rafts [110]. This CD95 compartmentalization contributes to reducing the apoptotic threshold leading to the clonotypic elimination of activated T-lymphocytes through activation of the CD95-mediated apoptotic signal [110]. Similarly, the reorganization of CD95 into DRMs can occur independent from ligand upon addition of certain chemotherapeutic drugs (e.g., rituximab [111], resveratrol [112, 113], edelfosine [87, 114, 115], aplidin [116], perifosine [115], cisplatin [117]). The molecular cascades that underlie this process remain elusive. Nevertheless, a growing body of evidence leads us to postulate that alteration of intracellular signaling pathway(s), such as the aforementioned PI3K signal [87, 90], may change biophysical properties of the plasma membrane, such as membrane fluidity, which in turn may facilitate CD95 clustering into large lipid raft-enriched platforms, favoring DISC formation and induction of the apoptotic program [118].

\subsubsection{Posttranslational Modifications}

Accumulation of CD95 mutations is not the only mechanism by which malignant cells inhibit the extrinsic signaling pathway. Posttranslational 
modifications in the intracellular tail of CD95, such as reversible oxidation or covalent attachment of a palmitic acid, were reported to alter the plasma membrane distribution of CD95 and thereby its subsequent signaling pathway. For instance, S-glutathionylation of mouse CD95 at cysteine 294 promotes clustering of CD95 and its distribution into lipid rafts [119]. This amino acid is conserved in the human CD95 sequence and corresponds to cysteine 304 (or C288 when subtraction of the 16-amino acid signal peptide is taken into consideration $[12,120])$. Interestingly, Janssen-Heininger and colleagues emphasize that death receptor glutathionylation occurs downstream of caspase- 8 and caspase- 3 activation whose catalytic activity damages the thioltransferase glutaredoxin 1 (Grx1), an enzyme implicated in the denitrosylation of proteins [119]. The consequence of Grx1 inactivation is the accumulation of glutathionylated CD95, which clusters into lipid rafts, sensitizing cells to the CD95-mediated apoptotic signal. Based on these findings, caspase- 8 activation occurs prior to aggregation of CD95 and redistribution into lipid rafts, both of which are requisite to form the DISC and subsequently activate larger amounts of caspase-8. In agreement with these observations, activation of caspase- 8 was reported to occur in a two-step process. That is, an immediate and small amount of activated caspase- $8(<1 \%)$ is generated when CD95L interacts with CD95 that orchestrates acid sphingomyelinase (ASM) activation, ceramide production, and CD95 clustering, which in turn promote DISC formation and the outburst of caspase- 8 processing essential to mount the apoptotic signal [121].

S-Glutathionylation consists in a bond between a reactive Cys-thiol and reduced glutathione (GSH), a tripeptide consisting of glycine, cysteine, and glutamate; its attachment to the protein will alter its structure and function in a manner similar to the addition of a phosphate [122]. S-Glutathionylation is not the only posttranslational modification of CD95 on a cysteine. S-Nitrosylation of cysteine 199 (corresponding to $\mathrm{C} 183$ after subtraction of signal peptide sequence) and 304 (C288) in colon and breast tumor cells also promotes the redistribution of
CD95 into DRMs, the formation of the DISC, and the transmission of the apoptotic signal [123].

Two reports have brought into light that covalent coupling of a 16-carbon fatty acid (palmitic acid) to cysteine 199 (C183) elicits the redistribution of CD95 into DRMs, the formation of SDS-stable CD95 microaggregates resistant to denaturing and reducing treatments, and the internalization of the receptor [124, 125]. Although their order remains to be fine-tuned, these molecular steps play a critical role in the implementation of apoptotic signals.

Of note, similar to S-nitrosylation, both the aforementioned S-glutathionylation at C304 (C288) and palmitoylation at C199 (C183) promote the partition of CD95 into lipid rafts and enhance the subsequent apoptotic signal. Further investigation is required to address whether these posttranslational modifications are redundant and occur simultaneously in dying cells or are elicited in a cell-specific and/or in a microenvironmentspecific manner. Understanding the molecular mechanisms controlling these posttranslational modifications would be of great interest in order to identify the mechanism by which tumor cells block them, leading to their resistance to the extrinsic signaling pathway.

\subsubsection{CD95 Internalization}

Using a powerful magnetic method to isolate receptor-containing endocytic vesicles, it has been shown that CD95 promptly associates with endosomal and lysosomal markers when incubated with an agonistic anti-CD95 mAb [126]. In addition, expression of a CD95 mutant in which the DD-located tyrosine 291 (Y275) is changed to phenylalanine does not seem to alter the capacity to bind FADD but compromises CD95L-mediated CD95 internalization occurring through an AP2/clathrin-driven endocytic pathway [126]. More strikingly, expression of the internalization-defective CD95 mutant Y291F abrogates the transmission of apoptotic signals, but fails to alter the non-apoptotic signaling pathways (i.e., NF-KB and ERK), and even promotes them (Fig. 11.3). These findings provide insight into the presence of a region in the DD, interacting with AP2 and promoting a clathrin-dependent 
endocytic pathway in a FADD-independent manner. Regarding the role of palmitoylation in receptor internalization, the interplay between lipid alteration and the AP2/clathrin-driven internalization of CD95 remains to be elucidated.

\subsubsection{4 $\mathrm{Ca}^{2+}$ Response}

It has been recently demonstrated that CD95 engagement evokes a rapid and transient $\mathrm{Ca}^{2+}$ signaling, which stimulates the recruitment of protein kinase C- $\beta 2$ (PKC- $\beta 2$ ) from the cytosol to the DISC [127]. This kinase transiently brakes DISC formation, providing a checkpoint before the irreversible commitment to cell death [128]. These findings raised the following questions: what are the $\mathrm{Ca}^{2+}$-dependent molecular mechanisms transiently inhibiting DISC formation, and do tumor cells use this signal to escape the immune response and/or resist chemotherapy?

\subsubsection{Programmed Necrosis also known as Necroptosis}

In 1998, inhibition of caspase activity was shown to sensitize fibroblastic L929 cell line to TNFmediated necrotic cell death [42]. With respect to CD95 signal, Tschopp et al. showed that FADD and RIP1 participate in the implementation of a non-apoptotic signaling pathway, which leads to a necrotic morphology without chromatin condensation and with loss of plasma membrane integrity [41]. Of note, BID cleavage was not observed in this necrotic signal. While FADD plays a crucial role in both apoptotic and necrotic pathways, RIP1 recruitment to CD95 occurs independently of this adaptor protein. Indeed, yeast two-hybrid experiments showed that RIP1 can bind directly to the CD95-DD, while this interaction is lost when a bait corresponding to mutated CD95-DD (replacement of Val 238 to Asn) is used [129]. In addition, RIP3 (RIPK3, a member of the RIP kinase family) is an indispensable factor for the induction of the necrotic signaling pathway [78-80]. A growing body of evidence supports the existence of necroptosis (programmed necrosis). In addition, identification of necrostatin, a chemical inhibitor of necroptosis [130], which specifically inhibits RIP1 kinase activity [131], has accelerated the pace of discovery in this field of cell death. Interplays exist between apoptosis and necroptosis; for instance, caspase-8, a potent inhibitor of necroptosis for both CD95 and TNFR1 [132], plays a critical role in necroptosis by its ability to process and inactivate RIP1 and RIP3 [133, 134]. At least for TNF signaling, the necrotic signal relies on the activity of CYLD, a deubiquitinating enzyme that is also cleaved and inactivated by caspase- 8 [135].

Overall, these findings suggest that the apoptotic machinery controls the necrotic one. This concept has been recently established in vivo by double-KO experiments [44-46, 136]. The KO of FADD or caspase- 8 is deleterious in mice mainly by the fact that these two apoptotic factors are beneficial in inhibiting a RIP1-/RIP3-dependent necrotic signal; thus, their loss unleashes the necroptotic program and leads to embryonic lethality. Yet, most studies on necroptosis have focused on the TNF signaling pathway, whereas the mechanism by which CD95 can elicit this cell death pathway, and how the switch in this receptor occurs between non-apoptotic, apoptotic, and necroptotic signals remains unclear. Importantly, the impact of each cell death on antigen presentation, and on the efficiency of immune response after elimination of infected or transformed cells, remains unclear.

\subsubsection{CD95L, an Inflammatory/ Oncogenic Cytokine?}

\subsubsection{A Ligand to Create Immune Privileges}

The transmembrane CD95L (CD178/FasL) is present at the surface of activated lymphocytes [64] and NK cells [137] where it orchestrates the elimination of transformed and infected cells. In addition, CD95L is expressed on the surface of neurons [138], corneal epithelia and endothelia [58, 139], and Sertoli cells [59] to prevent the infiltration of immune cells and thus to prohibit the spread of inflammation in these sensitive organs (i.e., brain, eyes, and testis, respectively), commonly called "immune-privileged" sites. 
The description of physiological immune privilege was followed by tumor-mediated immune privilege, since two groups reported that the ectopic expression of CD95L by malignant cells participated in the elimination of infiltrating T-lymphocytes and thus could play a role in the establishment of a tumor site whose access was denied to immune cells [140, 141]. However, these observations are controversial since ectopic expression of CD95L in allogenic transplant of $\beta$-islets [142, 143] and in tumor cell lines [144] led to a more rapid elimination of these cells than control cells, due to increased infiltration of neutrophils and macrophages endowed with antitumor activity.

\subsubsection{At Least Two Different Ligands and Two Different Signals}

Among the weapons at the disposal of immune cells, transmembrane CD95L contributes to the elimination of pre-tumor cells. Therefore, pretumor cells that escape the immunosurveillance will be shaped to develop resistance to CD95, a process termed immunoediting [145]. In other words, imprinting of the immune system on pre-tumor cells will select malignant cells with increased resistance toward the CD95L-induced signal. As previously mentioned, these alterations of the CD95 signal not only block the CD95mediated apoptotic signal but also promote the transmission of non-apoptotic signals by CD95L, which may play a critical role in carcinogenesis [106-108, 146]. In agreement with this hypothesis, a complete loss of CD95 expression is rarely observed in malignant cells [147].

Accumulating evidence indicates that the apoptotic ligand CD95L behaves as a chemoattractant for neutrophils, macrophages [50, 143, 144], T-lymphocytes [53], and malignant cells in which the CD95-mediated apoptotic signal is nonproductive [108, 148]. Nonetheless, the biological role of $\mathrm{CD} 95 \mathrm{~L}$ has to be clarified due to the fact that pathophysiologically the ligand is present in at least two forms with different stoichiometries. Indeed, CD95L is a transmembrane cytokine whose ectodomain can be cleaved by metalloproteases such as MMP3 [149], MMP7
[150], MMP9 [151], and ADAM-10 (a disintegrin and metalloproteinase 10) $[152,153]$ and released as a soluble ligand in the bloodstream. Based on the data demonstrating that a hexameric CD95L represents the minimal level of selfassociation required to signal apoptosis [154] and that cleavage by metalloproteases releases an homotrimeric ligand [154, 155], this soluble ligand has long been considered as an inert ligand competing with its membrane-bound counterpart for CD95 binding, thus acting as an antagonist of the death signal $[155,156]$. It has been recently demonstrated that this metalloprotease-cleaved CD95L (cl-CD95L) actively participates in the aggravation of inflammation and autoimmunity in patients affected by systemic lupus erythematosus (SLE) by inducing the non-apoptotic NF- $\kappa \mathrm{B}$ and PI3K [51, 53] signaling pathways (Fig. 11.4). Unlike transmembrane CD95L, induction of the PI3K signaling pathway by its metalloproteasecleaved counterpart occurs through the formation of a complex devoid of FADD and caspase-8 which recruits the src kinase c-yes instead [53, 148]; this unconventional receptosome was designated motility-inducing signaling complex (MISC) [53, 157] (Fig. 11.4). Even though experiments by the authors did not detect any trace of caspase- 8 in the MISC, this enzyme has been shown to participate in cell migration. The protease activity of caspase- 8 can be abolished by its phosphorylation at tyrosine 380 by src kinase [158]. This posttranslational modification was observed in cells stimulated with EGF and in colon cancer cells exhibiting constitutive activation of src; from a molecular standpoint, this modification does not alter caspase homodimerization or recruitment in DISC [158]. Moreover, the EGFR-driven phosphorylation of caspase-8 at Y380 turns out to be a potent inducer of the PI3K signaling pathway by recruiting the PI3K adaptor p85 alpha subunit [159]. Ultimately, caspase- 8 phosphorylation triggers cell migration. Nonetheless, it is noteworthy that CD95-induced migration and invasion do not appear to require an intact DD (reviewed in [160]), suggesting that either the caspase-8-dependent mode of cell migration occurs as an alternative signal for death receptors or that it only participates in non-death 
Pro-motile signaling pathway

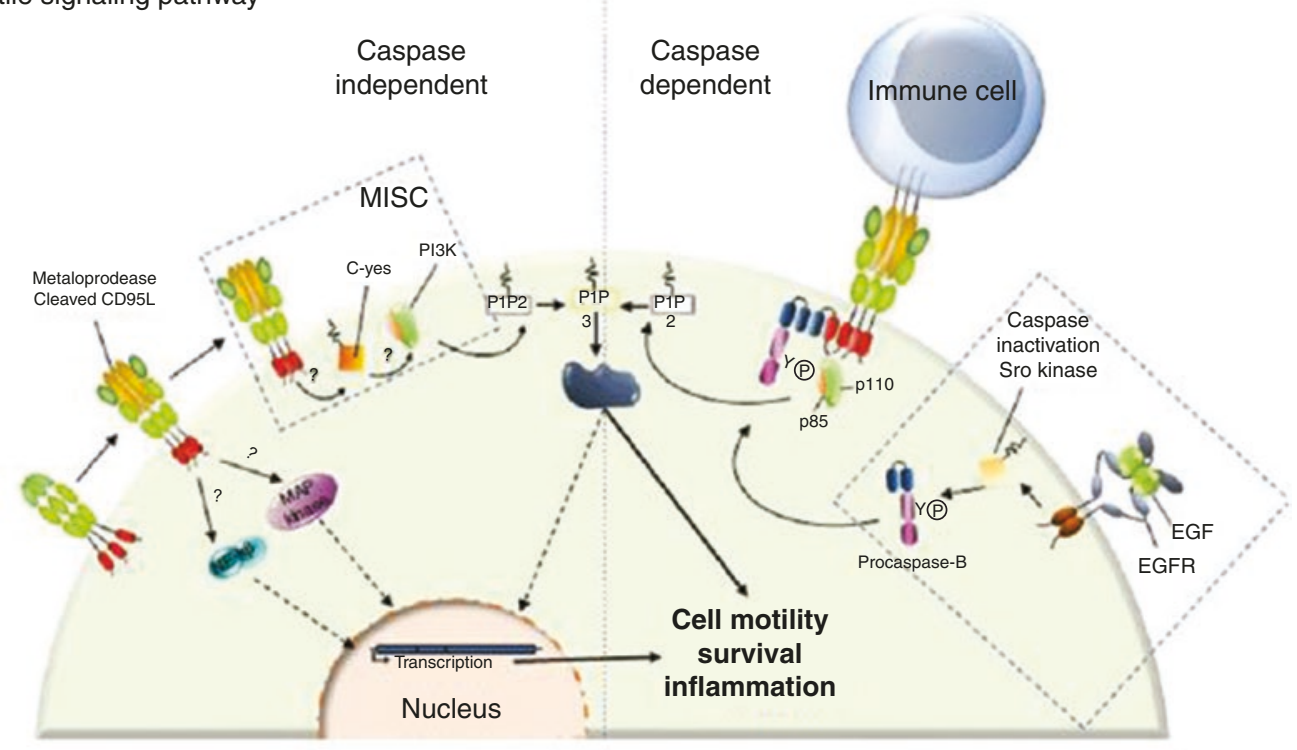

Fig. 11.4 CD95 triggers an unconventional PI3K signaling pathway. Left panel: In the presence of cl-CD95L, CD95 triggers MISC formation. This complex is devoid of FADD and caspase-8, but, instead, recruits the src kinase c-yes that implements the PI3K signaling pathway. CD95 engagement is also capable of NF- $\mathrm{KB}$ and MAPK activations through a yet unknown mechanism. Right

receptor-induced cell motility. It would be interesting to address this question in the future. To date, it can only be surmised that phosphorylation of caspase-8 at Y380 upon EGFR stimulation may prime certain cancer cells to become unresponsive to the apoptotic signal triggered by cytotoxic CD95L and meanwhile promote cell migration, an essential event in the course of cancer cell metastasis (Fig. 11.4).

It is noteworthy that in a similar manner, a decrease in the plasma membrane level of CD95 or expression of a mutated CD95 allele, as observed in ALPS patients and malignant cells, inhibits the implementation of the apoptotic signal but does not affect the transmission of non-apoptotic signals, such as NF-кB, MAPK, and PI3K [106, 107, 147], suggesting that these signals may stem from a different domain than CD95-DD or rely on different thresholds to be elicited. In summary, although the CD95/ CD95L interaction can eliminate malignant cells by implementation of the DISC or can pro- panel: It was reported that procaspase- 8 can be phosphorylated by the tyrosine kinase sre upon EGFR stimulation. This posttranslational modification not only blocks the catalytic activity of caspase- 8 but also promotes the recruitment of the p85 subunit of PI3K. We surmise that this caspase- 8 phosphorylation may favor the nonapoptotic signals induced by CD95

mote carcinogenesis by sustaining inflammation and/or by inducing metastatic dissemination $[50,51,53,108,147,148,161]$, the molecular mechanisms underlying the switch between these different signaling pathways remain enigmatic. An important question to be addressed is how the magnitude of CD95 aggregation controls the formation of "death"- vs. "motility"ISCs. Addressing these questions will lead to the development of new therapeutic agents with the ability to contain the spread of inflammation or impede carcinogenesis at least in pathologies involving increased soluble CD95L such as cancers (e.g., pancreatic cancer [162], large granular lymphocytic leukemia, breast cancer [157], and NK cell lymphoma [163]) or autoimmune disorders (e.g., rheumatoid arthritis and osteoarthritis [164], graft-versus-host-disease (GVHD) $[165,166]$, or SLE [53, 167]). Altogether, these studies support the notion that the death function of CD95 may correspond to its "day job," while the receptor may act as "a night killer" 
by fueling inflammation in certain pathophysiological contexts.

Strikingly, while the soluble form of CD95L generated by MMP7 (cleavage site inside the ${ }^{113} \mathrm{ELR}^{115}$ sequence, Fig. 11.5) induces apoptosis [150], its counterpart processed between serine 126 and leucine 127 does not [51, 53, 155]. To explain this discrepancy, one may speculate that the different quaternary structures of the naturally processed CD95L underlie the implementation of "death"- vs. "non-death"-inducing signaling complexes and downstream signals. In agreement with this notion, soluble CD95L bathed in the bronchoalveolar lavage (BALs) of patients suffering from acute respiratory distress syndrome (ARDS) undergoes oxidation at methionines 224 and 225 (Fig. 11.5), which enhances the aggregation level of the soluble ligand followed by its cytotoxic activity [168]. The same authors observed that the stalk region of CD95L, corresponding to amino acids 103-136 and encompassing the metalloprotease cleavage sites (Fig. 11.5), participates in the multimerization of CD95L, which accounts for the damage of the lung epithelium in ARDS [168]. Of note, in ARDS BALs, additional oxidation occurs at methionine 121 (Fig. 11.5), which in turn prevents the processing of CD95L by MMP7, and explains why this cytotoxic ligand keeps its stalk region [168]. Nonetheless, preservation of this region in soluble CD95L raises the question that whether an unidentified MMP7-independent cleavage site exists in the juxtamembrane region of CD95L, near the plasma membrane, or the ligand detected in ARDS patients corresponds to the full-length CD95L embedded in exosomes $[169,170]$. Indeed, this peculiar exosome-bound CD95L can be expressed by human prostate cancer cells (i.e., LNCaP) and evokes apoptosis in activated T-lymphocytes [171].

Overall, these findings emphasize that it will be of great interest in the future to finely characterize the quaternary structure of the naturally processed CD95L from the sera of patients affected by cancers or chronic/acute inflammatory disorders, to better understand the molecular mechanisms implemented by this ligand and thus predict its subsequent biological functions.

\subsection{Concluding Remarks}

Apoptosis is a fundamental process contributing to tissue homeostasis, immune response, and development. CD95, also called Fas, is a member of the tumor necrosis factor receptor (TNFR) superfamily. Its ligand, CD95L, was initially detected at the plasma membrane of activated T-lymphocytes and natural killer (NK) cells where it contributes to the elimination of transformed and infected cells. Given its implication in immune homeostasis and immune surveillance combined with the fact that

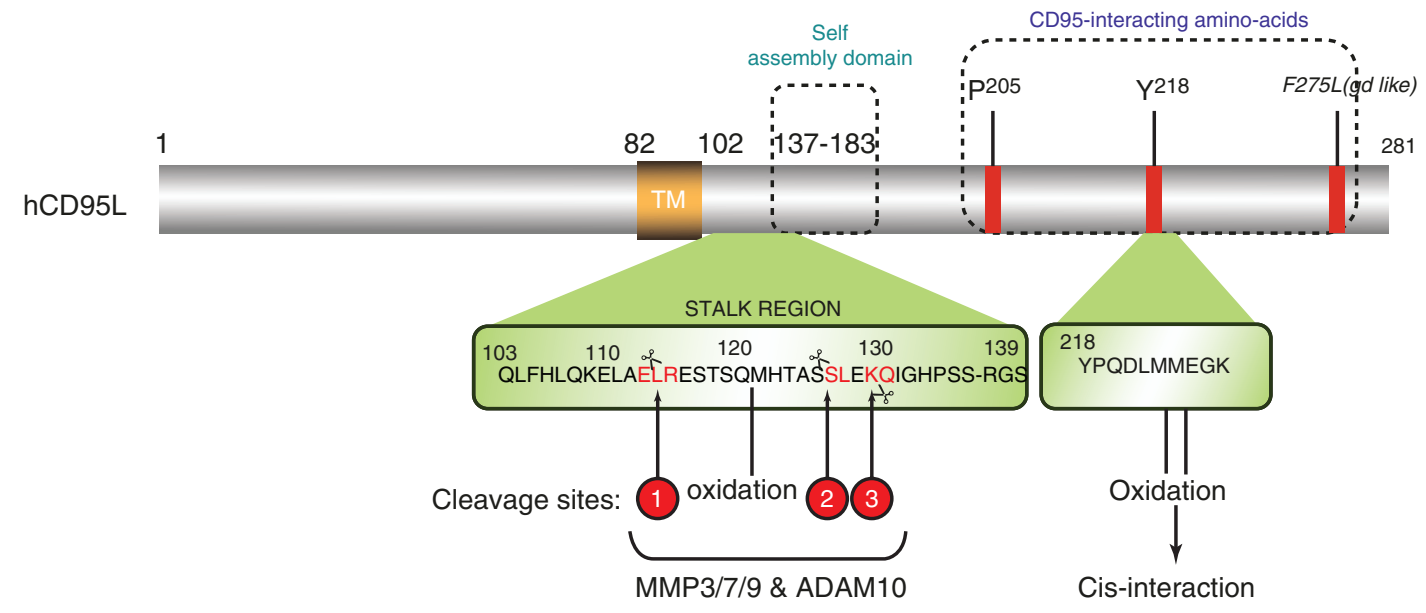

Fig. 11.5 CD95L: metalloprotease cleavage sites and domains 
various lineages of malignant cells exhibit lossof-function mutations, CD95 was initially classified as a tumor suppressor gene. Nonetheless, in different pathophysiological contexts, this receptor is able to transmit non-apoptotic signals and promote inflammation and carcinogenesis. Although the different non-apoptotic signaling pathways (NF- $\mathrm{BB}, \mathrm{MAPK}$, and PI3K) triggered by CD95 are known, the initial molecular events leading to these signals, the mechanisms by which the receptor switches from an apoptotic function to an inflammatory role, and, more importantly, the biological functions of these signals remain elusive.

\section{References}

1. Kerr JF, Wyllie AH, Currie AR. Apoptosis: a basic biological phenomenon with wide-ranging implications in tissue kinetics. $\mathrm{Br} \mathrm{J}$ Cancer. 1972;26(4):239-57.

2. Micheau O, Tschopp J. Induction of TNF receptor I-mediated apoptosis via two sequential signaling complexes. Cell. 2003;114(2):181-90.

3. Shimohama S. Apoptosis in Alzheimer's disease an update. Apoptosis. 2000;5(1):9-16.

4. Tatton WG. Apoptosis in Parkinson's disease: signals for neuronal degradation. Ann Neurol. 2003;53 Suppl 3:S61-70. discussion S70-2

5. Alnemri ES, et al. Human ICE/CED-3 protease nomenclature. Cell. 1996;87(2):171.

6. Hengartner MO. The biochemistry of apoptosis. Nature. 2000;407(6805):770-6.

7. Boldin MP, et al. A novel protein that interacts with the death domain of Fas/APO1 contains a sequence motif related to the death domain. J Biol Chem. 1995;270(14):7795-8.

8. Chinnaiyan AM, et al. FADD, a novel death domain-containing protein, interacts with the death domain of Fas and initiates apoptosis. Cell. 1995;81(4):505-12.

9. Hsu H, Xiong J, Goeddel DV. The TNF receptor 1-associated protein TRADD signals cell death and NF-kappa B activation. Cell. 1995;81(4):495-504.

10. Kischkel FC, et al. Cytotoxicity-dependent APO-1 (Fas/CD95)-associated proteins form a deathinducing signaling complex (DISC) with the receptor. EMBO J. 1995;14(22):5579-88.

11. Li P, et al. Cytochrome $\mathrm{c}$ and dATP-dependent formation of Apaf-1/caspase-9 complex initiates an apoptotic protease cascade. Cell. 1997;91(4):479-89.

12. Itoh $\mathrm{N}$, et al. The polypeptide encoded by the cDNA for human cell surface antigen Fas can mediate apoptosis. Cell. 1991;66(2):233-43.
13. Loetscher $\mathrm{H}$, et al. Molecular cloning and expression of the human $55 \mathrm{kd}$ tumor necrosis factor receptor. Cell. 1990;61(2):351-9.

14. Pan G, et al. The receptor for the cytotoxic ligand TRAIL. Science. 1997;276(5309):111-3.

15. Walczak H, et al. TRAIL-R2: a novel apoptosismediating receptor for TRAIL. EMBO J. 1997;16(17):5386-97.

16. Pan $\mathrm{G}$, et al. Identification and functional characterization of DR6, a novel death domain-containing TNF receptor. FEBS Lett. 1998;431(3):351-6.

17. Alderson MR, et al. Fas transduces activation signals in normal human T lymphocytes. J Exp Med. 1993;178(6):2231-5.

18. Schulze-Osthoff K, Krammer PH, Droge W. Divergent signalling via APO-1/Fas and the TNF receptor, two homologous molecules involved in physiological cell death. EMBO J. 1994;13(19):4587-96.

19. Smith CA, Farrah T, Goodwin RG. The TNF receptor superfamily of cellular and viral proteins: activation, costimulation, and death. Cell. 1994;76(6):959-62.

20. Locksley RM, Killeen N, Lenardo MJ. The TNF and TNF receptor superfamilies: integrating mammalian biology. Cell. 2001;104(4):487-501.

21. Bodmer JL, Schneider P, Tschopp J. The molecular architecture of the TNF superfamily. Trends Biochem Sci. 2002;27(1):19-26.

22. Edmond V, et al. Precise mapping of the CD95 pre-ligand assembly domain. PLoS One. 2012;7(9):e46236.

23. Papoff G, et al. Identification and characterization of a ligand-independent oligomerization domain in the extracellular region of the CD95 death receptor. J Biol Chem. 1999;274(53):38241-50.

24. Siegel RM, et al. Fas preassociation required for apoptosis signaling and dominant inhibition by pathogenic mutations. Science. 2000;288(5475):2354-7.

25. Itoh N, Nagata $\mathrm{S}$. A novel protein domain required for apoptosis. Mutational analysis of human Fas antigen. J Biol Chem. 1993;268(15):10932-7.

26. Tartaglia LA, et al. A novel domain within the $55 \mathrm{kd}$ TNF receptor signals cell death. Cell. 1993;74(5):845-53.

27. Tang $\mathrm{W}$, et al. The growth factor progranulin binds to TNF receptors and is therapeutic against inflammatory arthritis in mice. Science. 2011;332(6028):478-84.

28. Cabal-Hierro L, Lazo PS. Signal transduction by tumor necrosis factor receptors. Cell Signal. 2012;24(6):1297-305.

29. Chan FK, et al. A domain in TNF receptors that mediates ligand-independent receptor assembly and signaling. Science. 2000;288(5475):2351-4.

30. Pennica D, et al. Human tumour necrosis factor: precursor structure, expression and homology to lymphotoxin. Nature. 1984;312(5996):724-9.

31. Black RA, et al. A metalloproteinase disintegrin that releases tumour-necrosis factor-alpha from cells. Nature. 1997;385(6618):729-33. 
32. Moss ML, et al. Cloning of a disintegrin metalloproteinase that processes precursor tumour-necrosis factor-alpha. Nature. 1997;385(6618):733-6.

33. Grell M, et al. The transmembrane form of tumor necrosis factor is the prime activating ligand of the $80 \mathrm{kDa}$ tumor necrosis factor receptor. Cell. 1995;83(5):793-802.

34. Wang CY, et al. NF-kappaB antiapoptosis: induction of TRAF1 and TRAF2 and c-IAP1 and c-IAP2 to suppress caspase-8 activation. Science. 1998;281(5383):1680-3.

35. Haas TL, et al. Recruitment of the linear ubiquitin chain assembly complex stabilizes the TNF-R1 signaling complex and is required for TNF-mediated gene induction. Mol Cell. 2009;36(5):831-44.

36. Kirisako T, et al. A ubiquitin ligase complex assembles linear polyubiquitin chains. EMBO J. 2006;25(20):4877-87.

37. Gerlach B, et al. Linear ubiquitination prevents inflammation and regulates immune signalling. Nature. 2011;471(7340):591-6.

38. Poukkula M, et al. Rapid turnover of c-FLIPshort is determined by its unique C-terminal tail. J Biol Chem. 2005;280(29):27345-55.

39. Enesa K, et al. NF-kappaB suppression by the deubiquitinating enzyme Cezanne: a novel negative feedback loop in pro-inflammatory signaling. J Biol Chem. 2008;283(11):7036-45.

40. Green DR, et al. RIPK-dependent necrosis and its regulation by caspases: a mystery in five acts. Mol Cell. 2011;44(1):9-16.

41. Holler N, et al. Fas triggers an alternative, caspase-8independent cell death pathway using the kinase RIP as effector molecule. Nat Immunol. 2000;1(6):489-95.

42. Vercammen D, et al. Inhibition of caspases increases the sensitivity of L929 cells to necrosis mediated by tumor necrosis factor. J Exp Med. 1998;187(9):1477-85.

43. Cho YS, et al. Phosphorylation-driven assembly of the RIP1-RIP3 complex regulates programmed necrosis and virus-induced inflammation. Cell. 2009;137(6):1112-23.

44. Kaiser WJ, et al. RIP3 mediates the embryonic lethality of caspase-8-deficient mice. Nature. 2011;471(7338):368-72.

45. Oberst A, et al. Catalytic activity of the caspase-8FLIP(L) complex inhibits RIPK3-dependent necrosis. Nature. 2011;471(7338):363-7.

46. Welz PS, et al. FADD prevents RIP3-mediated epithelial cell necrosis and chronic intestinal inflammation. Nature. 2011;477(7364):330-4.

47. Feldmann M, Maini RN. Lasker clinical medical research award TNF defined as a therapeutic target for rheumatoid arthritis and other autoimmune diseases. Nat Med. 2003;9(10):1245-50.

48. Desbarats J, et al. Fas engagement induces neurite growth through ERK activation and p35 upregulation. Nat Cell Biol. 2003;5(2):118-25.

49. Desbarats J, Newell MK. Fas engagement accelerates liver regeneration after partial hepatectomy. Nat Med. 2000;6(8):920-3.
50. Letellier E, et al. CD95-ligand on peripheral myeloid cells activates Syk kinase to trigger their recruitment to the inflammatory site. Immunity. 2010;32(2):240-52.

51. O'Reilly LA, et al. Membrane-bound Fas ligand only is essential for Fas-induced apoptosis. Nature. 2009;461(7264):659-63.

52. Ruan W, Lee CT, Desbarats J. A novel juxtamembrane domain in tumor necrosis factor receptor superfamily molecules activates Rac1 and controls neurite growth. Mol Biol Cell. 2008;19(8):3192-202.

53. Tauzin $\mathrm{S}$, et al. The naturally processed CD95L elicits a c-yes/calcium/PI3K-driven cell migration pathway. PLoS Biol. 2011;9(6):e1001090.

54. Tauzin S, et al. CD95-mediated cell signaling in cancer: mutations and post-translational modulations. Cell Mol Life Sci. 2012;69(8):1261-77.

55. Trauth BC, et al. Monoclonal antibody-mediated tumor regression by induction of apoptosis. Science. 1989;245(4915):301-5.

56. Suda T, et al. Molecular cloning and expression of the Fas ligand, a novel member of the tumor necrosis factor family. Cell. 1993;75(6):1169-78.

57. Oshimi Y, et al. Involvement of Fas ligand and Fasmediated pathway in the cytotoxicity of human natural killer cells. J Immunol. 1996;157(7):2909-15.

58. Griffith TS, et al. Fas ligand-induced apoptosis as a mechanism of immune privilege. Science. 1995;270(5239):1189-92.

59. Bellgrau D, et al. A role for CD95 ligand in preventing graft rejection. Nature. 1995;377(6550):630-2.

60. Watanabe-Fukunaga $R$, et al. Lymphoproliferation disorder in mice explained by defects in Fas antigen that mediates apoptosis. Nature. 1992;356(6367):314-7.

61. Adachi M, Watanabe-Fukunaga R, Nagata S. Aberrant transcription caused by the insertion of an early transposable element in an intron of the Fas antigen gene of lpr mice. Proc Natl Acad Sci U S A. 1993;90(5):1756-60.

62. Chu JL, et al. The defect in Fas mRNA expression in MRL/lpr mice is associated with insertion of the retrotransposon, ETn. J Exp Med. 1993;178(2):723-30.

63. Kimura M, Matsuzawa A. Autoimmunity in mice bearing lprcg: a novel mutant gene. Int Rev Immunol. 1994;11(3):193-210.

64. Takahashi T, et al. Generalized lymphoproliferative disease in mice, caused by a point mutation in the Fas ligand. Cell. 1994;76(6):969-76.

65. Strasser A, Jost PJ, Nagata S. The many roles of FAS receptor signaling in the immune system. Immunity. 2009;30(2):180-92.

66. Behrmann I, Walczak H, Krammer PH. Structure of the human APO-1 gene. Eur J Immunol. 1994;24(12):3057-62.

67. Huang B, et al. NMR structure and mutagenesis of the Fas (APO-1/CD95) death domain. Nature. 1996;384(6610):638-41.

68. Scott FL, et al. The Fas-FADD death domain complex structure unravels signalling by receptor clustering. Nature. 2009;457(7232):1019-22. 
69. Esposito D, et al. Solution NMR investigation of the CD95/FADD homotypic death domain complex suggests lack of engagement of the CD95 C terminus. Structure. 2010;18(10):1378-90.

70. Wang L, et al. The Fas-FADD death domain complex structure reveals the basis of DISC assembly and disease mutations. Nat Struct Mol Biol. 2010;17(11):1324-9.

71. Muppidi JR, et al. Homotypic FADD interactions through a conserved RXDLL motif are required for death receptor-induced apoptosis. Cell Death Differ. 2006;13(10):1641-50.

72. Irmler $\mathrm{M}$, et al. Inhibition of death receptor signals by cellular FLIP. Nature. 1997;388(6638):190-5.

73. Thome M, et al. Viral FLICE-inhibitory proteins (FLIPs) prevent apoptosis induced by death receptors. Nature. 1997;386(6624):517-21.

74. Condorelli G, et al. PED/PEA-15: an anti-apoptotic molecule that regulates FAS/TNFR1-induced apoptosis. Oncogene. 1999;18(31):4409-15.

75. Scaffidi C, et al. Two CD95 (APO-1/Fas) signaling pathways. EMBO J. 1998;17(6):1675-87.

76. Algeciras-Schimnich A, et al. Two CD95 tumor classes with different sensitivities to antitumor drugs. Proc Natl Acad Sci U S A. 2003;100(20):11445-50.

77. Chaigne-Delalande B, et al. CD95 engagement mediates actin-independent and -dependent apoptotic signals. Cell Death Differ. 2009;16(12):1654-64.

78. Yin XM. Signal transduction mediated by bid, a pro-death Bcl-2 family proteins, connects the death receptor and mitochondria apoptosis pathways. Cell Res. 2000;10(3):161-7.

79. Yin XM, et al. Bid-deficient mice are resistant to Fas-induced hepatocellular apoptosis. Nature. 1999;400(6747):886-91.

80. Jost PJ, et al. XIAP discriminates between type I and type II FAS-induced apoptosis. Nature. 2009;460(7258):1035-9.

81. Roy N, et al. The c-IAP-1 and c-IAP-2 proteins are direct inhibitors of specific caspases. EMBO J. 1997;16(23):6914-25.

82. Deveraux QL, et al. X-linked IAP is a direct inhibitor of cell-death proteases. Nature. 1997;388(6639):300-4.

83. Deveraux QL, et al. IAPs block apoptotic events induced by caspase- 8 and cytochrome $\mathrm{c}$ by direct inhibition of distinct caspases. EMBO J. 1998;17(8):2215-23.

84. Suzuki Y, Nakabayashi Y, Takahashi R. Ubiquitinprotein ligase activity of X-linked inhibitor of apoptosis protein promotes proteasomal degradation of caspase- 3 and enhances its anti-apoptotic effect in Fas-induced cell death. Proc Natl Acad Sci U S A. 2001;98(15):8662-7.

85. Du C, et al. Smac, a mitochondrial protein that promotes cytochrome c-dependent caspase activation by eliminating IAP inhibition. Cell. 2000;102(1):33-42.

86. Sun XM, et al. Bcl-2 and Bcl-xL inhibit CD95mediated apoptosis by preventing mitochondrial release of Smac/DIABLO and subsequent inactiva- tion of X-linked inhibitor-of-apoptosis protein. J Biol Chem. 2002;277(13):11345-51.

87. Beneteau M, et al. Localization of Fas/CD95 into the lipid rafts on down-modulation of the phosphatidylinositol 3-kinase signaling pathway. Mol Cancer Res. 2008;6(4):604-13.

88. Peacock JW, et al. PTEN loss promotes mitochondrially dependent type II Fas-induced apoptosis via PEA-15. Mol Cell Biol. 2009;29(5):1222-34.

89. Varadhachary AS, et al. Phosphatidylinositol 3'-kinase blocks CD95 aggregation and caspase-8 cleavage at the death-inducing signaling complex by modulating lateral diffusion of CD95. J Immunol. 2001;166(11):6564-9.

90. Pizon M, et al. Actin-independent exclusion of CD95 by PI3K/AKT signalling: implications for apoptosis. Eur J Immunol. 2011;41(8):2368-78.

91. Renganathan $\mathrm{H}$, et al. Phosphorylation of PEA-15 switches its binding specificity from ERK/MAPK to FADD. Biochem J. 2005;390(Pt 3):729-35.

92. Trencia A, et al. Protein kinase B/Akt binds and phosphorylates PED/PEA-15, stabilizing its antiapoptotic action. Mol Cell Biol. 2003;23(13):4511-21.

93. Strasser A, et al. Bcl-2 and Fas/APO-1 regulate distinct pathways to lymphocyte apoptosis. EMBO J. 1995;14(24):6136-47.

94. Lacronique $\mathrm{V}$, et al. Bcl-2 protects from lethal hepatic apoptosis induced by an anti-Fas antibody in mice. Nat Med. 1996;2(1):80-6.

95. Rodriguez I, et al. A bcl-2 transgene expressed in hepatocytes protects mice from fulminant liver destruction but not from rapid death induced by anti-Fas antibody injection. $\mathrm{J}$ Exp Med. 1996;183(3):1031-6.

96. Drappa J, et al. Fas gene mutations in the CanaleSmith syndrome, an inherited lymphoproliferative disorder associated with autoimmunity. N Engl J Med. 1996;335(22):1643-9.

97. Fisher GH, et al. Dominant interfering Fas gene mutations impair apoptosis in a human autoimmune lymphoproliferative syndrome. Cell. 1995;81(6):935-46.

98. Rieux-Laucat F, et al. Mutations in Fas associated with human lymphoproliferative syndrome and autoimmunity. Science. 1995;268(5215):1347-9.

99. Canale VC, Smith CH. Chronic lymphadenopathy simulating malignant lymphoma. J Pediatr. 1967;70(6):891-9.

100. Rieux-Laucat F, et al. Lymphoproliferative syndrome with autoimmunity: a possible genetic basis for dominant expression of the clinical manifestations. Blood. 1999;94(8):2575-82.

101. Straus SE, et al. The development of lymphomas in families with autoimmune lymphoproliferative syndrome with germline Fas mutations and defective lymphocyte apoptosis. Blood. 2001;98(1):194-200.

102. Hennino A, et al. FLICE-inhibitory protein is a key regulator of germinal center B cell apoptosis. J Exp Med. 2001;193(4):447-58. 
103. Montesinos-Rongen M, et al. Primary diffuse large B-cell lymphomas of the central nervous system are targeted by aberrant somatic hypermutation. Blood. 2004;103(5):1869-75.

104. Muschen M, et al. The origin of CD95-gene mutations in B-cell lymphoma. Trends Immunol. 2002;23(2):75-80.

105. Peter ME, Legembre P, Barnhart BC. Does CD95 have tumor promoting activities? Biochim Biophys Acta. 2005;1755(1):25-36.

106. Legembre P, Barnhart BC, Peter ME. The relevance of NF-kappaB for CD95 signaling in tumor cells. Cell Cycle. 2004;3(10):1235-9.

107. Legembre P, et al. Induction of apoptosis and activation of NF-kappaB by CD95 require different signalling thresholds. EMBO Rep. 2004;5(11):1084-9.

108. Barnhart BC, et al. CD95 ligand induces motility and invasiveness of apoptosis-resistant tumor cells. EMBO J. 2004;23(15):3175-85.

109. Grassme H, et al. CD95 signaling via ceramide-rich membrane rafts. J Biol Chem. 2001;276(23):20589-96.

110. Muppidi JR, Siegel RM. Ligand-independent redistribution of Fas (CD95) into lipid rafts mediates clonotypic T cell death. Nat Immunol. 2004;5(2):182-9.

111. Stel AJ, et al. Fas receptor clustering and involvement of the death receptor pathway in rituximabmediated apoptosis with concomitant sensitization of lymphoma B cells to fas-induced apoptosis. J Immunol. 2007;178(4):2287-95.

112. Delmas D, et al. Resveratrol-induced apoptosis is associated with Fas redistribution in the rafts and the formation of a death-inducing signaling complex in colon cancer cells. J Biol Chem. 2003;278(42):41482-90.

113. Delmas D, et al. Redistribution of CD95, DR4 and DR5 in rafts accounts for the synergistic toxicity of resveratrol and death receptor ligands in colon carcinoma cells. Oncogene. 2004;23(55):8979-86.

114. Gajate C, et al. Intracellular triggering of Fas aggregation and recruitment of apoptotic molecules into Fas-enriched rafts in selective tumor cell apoptosis. J Exp Med. 2004;200(3):353-65.

115. Gajate C, Mollinedo F. Edelfosine and perifosine induce selective apoptosis in multiple myeloma by recruitment of death receptors and downstream signaling molecules into lipid rafts. Blood. 2007;109(2):711-9.

116. Gajate C, Mollinedo F. Cytoskeleton-mediated death receptor and ligand concentration in lipid rafts forms apoptosis-promoting clusters in cancer chemotherapy. J Biol Chem. 2005;280(12):11641-7.

117. Lacour S, et al. Cisplatin-induced CD95 redistribution into membrane lipid rafts of HT29 human colon cancer cells. Cancer Res. 2004;64(10):3593-8.

118. Segui B, Legembre P. Redistribution of CD95 into the lipid rafts to treat cancer cells? Recent Pat Anticancer Drug Discov. 2010;5(1):22-8.

119. Anathy V, et al. Redox amplification of apoptosis by caspase-dependent cleavage of glutaredoxin
1 and S-glutathionylation of Fas. J Cell Biol. 2009; 184(2):241-52.

120. Oehm A, et al. Purification and molecular cloning of the APO-1 cell surface antigen, a member of the tumor necrosis factor/nerve growth factor receptor superfamily. Sequence identity with the Fas antigen. J Biol Chem. 1992;267(15):10709-15.

121. Grassme $\mathrm{H}$, et al. Ceramide-mediated clustering is required for CD95-DISC formation. Oncogene. 2003;22(35):5457-70.

122. Chen CA, et al. S-glutathionylation uncouples eNOS and regulates its cellular and vascular function. Nature. 2010;468(7327):1115-8.

123. Leon-Bollotte L, et al. S-nitrosylation of the death receptor fas promotes fas ligand-mediated apoptosis in cancer cells. Gastroenterology. 2011;140(7):2009_ 18,2018 e1-4.

124. Chakrabandhu $\mathrm{K}$, et al. Palmitoylation is required for efficient Fas cell death signaling. EMBO J. 2007;26(1):209-20.

125. Feig C, et al. Palmitoylation of CD95 facilitates formation of SDS-stable receptor aggregates that initiate apoptosis signaling. EMBO J. 2007;26(1):221-31.

126. Lee $\mathrm{KH}$, et al. The role of receptor internalization in CD95 signaling. EMBO J. 2006;25(5):1009-23.

127. Khadra N, et al. CD95 triggers Orai1-mediated localized $\mathrm{Ca} 2+$ entry, regulates recruitment of protein kinase $\mathrm{C}$ (PKC) beta2, and prevents death-inducing signaling complex formation. Proc Natl Acad Sci U S A. 2011;108(47):19072-7.

128. Penna A, et al. The CD95 signaling pathway: to not die and fly. Commun Integr Biol. 2012;5(2):190-2.

129. Stanger BZ, et al. RIP: a novel protein containing a death domain that interacts with Fas/APO-1 (CD95) in yeast and causes cell death. Cell. 1995;81(4):513-23.

130. Degterev A, et al. Chemical inhibitor of nonapoptotic cell death with therapeutic potential for ischemic brain injury. Nat Chem Biol. 2005;1(2):112-9.

131. Degterev A, et al. Identification of RIP1 kinase as a specific cellular target of necrostatins. Nat Chem Biol. 2008;4(5):313-21.

132. Lee EW, et al. The roles of FADD in extrinsic apoptosis and necroptosis. BMB Rep. 2012;45(9):496-508.

133. Lin Y, et al. Cleavage of the death domain kinase RIP by caspase- 8 prompts TNF-induced apoptosis. Genes Dev. 1999;13(19):2514-26.

134. Feng S, et al. Cleavage of RIP3 inactivates its caspase-independent apoptosis pathway by removal of kinase domain. Cell Signal. 2007;19(10):2056-67.

135. O'Donnell MA, et al. Caspase 8 inhibits programmed necrosis by processing CYLD. Nat Cell Biol. 2011;13(12):1437-42.

136. Peter ME. Programmed cell death: apoptosis meets necrosis. Nature. 2011;471(7338):310-2.

137. Montel AH, et al. Fas involvement in cytotoxicity mediated by human NK cells. Cell Immunol. 1995;166(2):236-46. 
138. Saas P, et al. Fas ligand expression by astrocytoma in vivo: maintaining immune privilege in the brain? J Clin Invest. 1997;99(6):1173-8.

139. Stuart PM, et al. CD95 ligand (FasL)-induced apoptosis is necessary for corneal allograft survival. J Clin Invest. 1997;99(3):396-402.

140. Hahne M, et al. Melanoma cell expression of Fas(Apo-1/CD95) ligand: implications for tumor immune escape. Science. 1996;274(5291):1363-6.

141. O'Connell J, et al. The Fas counterattack: Fasmediated T cell killing by colon cancer cells expressing Fas ligand. J Exp Med. 1996;184(3):1075-82.

142. Allison J, et al. Transgenic expression of CD95 ligand on islet beta cells induces a granulocytic infiltration but does not confer immune privilege upon islet allografts. Proc Natl Acad Sci U S A. 1997;94(8):3943-7.

143. Kang SM, et al. Fas ligand expression in islets of Langerhans does not confer immune privilege and instead targets them for rapid destruction. Nat Med. 1997;3(7):738-43.

144. Chen JJ, Sun Y, Nabel GJ. Regulation of the proinflammatory effects of Fas ligand (CD95L). Science. 1998;282(5394):1714-7.

145. Bui JD, Schreiber RD. Cancer immunosurveillance, immunoediting and inflammation: independent or interdependent processes? Curr Opin Immunol. 2007;19(2):203-8.

146. Beneteau M, et al. Dominant-negative Fas mutation is reversed by down-expression of c-FLIP. Cancer Res. 2007;67(1):108-15.

147. Chen L, et al. CD95 promotes tumour growth. Nature. 2010;465(7297):492-6.

148. Kleber S, et al. Yes and PI3K bind CD95 to signal invasion of glioblastoma. Cancer Cell. 2008;13(3):235-48.

149. Matsuno H, et al. Stromelysin-1 (MMP-3) in synovial fluid of patients with rheumatoid arthritis has potential to cleave membrane bound Fas ligand. J Rheumatol. 2001;28(1):22-8.

150. Vargo-Gogola T, et al. Identification of novel matrix metalloproteinase-7 (matrilysin) cleavage sites in murine and human Fas ligand. Arch Biochem Biophys. 2002;408(2):155-61.

151. Kiaei M, et al. Matrix metalloproteinase-9 regulates TNF-alpha and FasL expression in neuronal, glial cells and its absence extends life in a transgenic mouse model of amyotrophic lateral sclerosis. Exp Neurol. 2007;205(1):74-81.

152. Kirkin V, et al. The Fas ligand intracellular domain is released by ADAM10 and SPPL2a cleavage in T-cells. Cell Death Differ. 2007;14(9):1678-87.

153. Schulte M, et al. ADAM10 regulates FasL cell surface expression and modulates FasL-induced cytotoxicity and activation-induced cell death. Cell Death Differ. 2007;14(5):1040-9.

154. Holler N, et al. Two adjacent trimeric Fas ligands are required for Fas signaling and formation of a death-inducing signaling complex. Mol Cell Biol. 2003;23(4):1428-40.
155. Schneider $\mathrm{P}$, et al. Conversion of membranebound Fas(CD95) ligand to its soluble form is associated with downregulation of its proapoptotic activity and loss of liver toxicity. J Exp Med. 1998;187(8):1205-13.

156. Suda T, et al. Membrane Fas ligand kills human peripheral blood $\mathrm{T}$ lymphocytes, and soluble Fas ligand blocks the killing. J Exp Med. 1997;186(12):2045-50.

157. Malleter $\mathrm{M}$, et al. CD95L cell surface cleavage triggers a pro-metastatic signaling pathway in triple negative breast cancer. Cancer Res. 2013;73(22):6711-21.

158. Cursi S, et al. Src kinase phosphorylates Caspase-8 on Tyr380: a novel mechanism of apoptosis suppression. EMBO J. 2006;25(9):1895-905.

159. Senft J, Helfer B, Frisch SM. Caspase-8 interacts with the p85 subunit of phosphatidylinositol 3-kinase to regulate cell adhesion and motility. Cancer Res. 2007;67(24):11505-9.

160. Steller EJ, Borel Rinkes IH, Kranenburg O. How CD95 stimulates invasion. Cell Cycle. 2011;10(22):3857-62.

161. Bivona TG, et al. FAS and NF-kappaB signalling modulate dependence of lung cancers on mutant EGFR. Nature. 2011;471(7339):523-6.

162. Bellone G, et al. Production and pro-apoptotic activity of soluble CD95 ligand in pancreatic carcinoma. Clin Cancer Res. 2000;6(6):2448-55.

163. Tanaka M, et al. Fas ligand in human serum. Nat Med. 1996;2(3):317-22.

164. Hashimoto H, et al. Soluble Fas ligand in the joints of patients with rheumatoid arthritis and osteoarthritis. Arthritis Rheum. 1998;41(4):657-62.

165. Das H, et al. Levels of soluble FasL and FasL gene expression during the development of graft-versushost disease in DLT-treated patients. Br J Haematol. 1999;104(4):795-800.

166. Kanda Y, et al. Increased soluble Fas-ligand in sera of bone marrow transplant recipients with acute graft-versus-host disease. Bone Marrow Transplant. 1998;22(8):751-4.

167. Tomokuni A, et al. Serum levels of soluble Fas ligand in patients with silicosis. Clin Exp Immunol. 1999;118(3):441-4.

168. Herrero R, et al. The biological activity of FasL in human and mouse lungs is determined by the structure of its stalk region. J Clin Invest. 2011;121(3):1174-90.

169. Alonso R, et al. Diacylglycerol kinase alpha regulates the formation and polarisation of mature multivesicular bodies involved in the secretion of Fas ligand-containing exosomes in T lymphocytes. Cell Death Differ. 2011;18(7):1161-73.

170. Bianco NR, et al. Modulation of the immune response using dendritic cell-derived exosomes. Methods Mol Biol. 2007;380:443-55.

171. Abusamra AJ, et al. Tumor exosomes expressing Fas ligand mediate CD8+ T-cell apoptosis. Blood Cells Mol Dis. 2005;35(2):169-73. 